\title{
COMMISSION 42: CLOSE BINARY STARS
}

\section{(ETOILES BINAIRES SERREES)}

\author{
PRESIDENT: Paula Szkody \\ VICE-PRESIDENT: Alvaro Giménez \\ ORGANIZING COMMITTEE: E.F. Guinan, L. Bianchi, H. Drechsel, \\ D. Gies, Y-W. Kang, J. Linsky, M. Livio, N. Morrell, V. Niemela, Y. Osaki, \\ S. Rucinski, C. Sterken \& J.H. Wood
}

\section{Introduction}

It has been a great pleasure to work with the members of $\mathrm{C} 42$ in a field which is so active in pursuing the latest technology and new ideas. This activity allows continual progress toward understanding the physical parameters and evolutionary consequences of close binary membership. With the launch of XMM, Chandra and FUSE all in 1999, the increased sensitivity and especially resolution in the X-ray and ultraviolet regimes have led to tremendous advances in our knowledge of the hot components in close binaries. For those systems undergoing active accretion, we are able to determine, for the first time, the temperature, velocity and compositions of the accretion areas. At the same time, the first results from the largest ground-based telescopes (VLT, Gemini, HET, MMT) are pushing back the limits of the faintest and farthest systems that can be studied, as well as improving the time-resolution of the data on nearby systems. In addition, large scale surveys (HQS, 2dF, SDSS, FSVS, OGLE) are finding large numbers of new, interesting close binary systems which may alter previous density estimates that were based on selection effects geared to high luminosity systems and which provide us with a huge supply of objects to pursue.

Due to the general web access of ADS and SIMBAD and the summary of papers on close binary research contained in the Bibliography of Close Binaries (BCB), accessed from the $\mathrm{C} 42$ web site www.astro.washington.edu/szkody/c42/, this brief triennial report is used to merely highlight the major contributions to our field. In addition, I would like to thank a few individuals who have performed exceptional service for C42. This includes Colin Scarfe, who has taken on the responsibilites of Editor-in-Chief of BCB after the retirement of Horst Drechsel, and along with the editorial board consisting of H. Drechsel, D. R. Faulkner, V. G. Karetnikov, E. Lapasset, C. Maceroni, Y. Nakamura, P. G. Niarchos, R. G. Samec, W. Van Hamme and M. Wolf, have accomplished a seamless transition and moved to pure electronic access. We are also grateful for the work of Co-Editors Katalin Oláh and J. Jurcsik for the IBVS (accessible also through the C42 web site) and its editorial board (chair, Don Kurtz) for their continuing production of this publication for results in our field. Lastly, I would like to thank the OC members of $\mathrm{C} 42$ for their advice and participation in setting up the Colloquia, Symposia and JDs associated with the Sydney meeting and throughout the past 3 years.

\section{Conferences and Books}

As usual, C42 members had many meetings to discuss current and future research and the resulting conference proceedings serve as the best summaries of the important research in each specialized area. The meetings with a large part devoted to close binaries include: The Influence of Binaries on Stellar Population Studies, Brussels, 2000, (Kluwer); Evolution of Binary and Multiple Star Systems, Bormio, 2000, (ASP Conf. Ser. 22); 
The Physics of Cataclysmic Variables and Related Objects, Göttingen, 2001, (ASP Conf. Ser. 261); Variable Stars, Odessa, 2001; Cool Stars, Stellar Systems and the Sun, 11th \& 12th Cambridge Workshops, Tenerife, 1999, and Boulder, 2001, (ASP Conf. Ser. 223 and http://origins.Colorado.edu/cs12/); Continuing the Challenge of EUV Astronomy: Current Analysis and Prospects for the Future, Jenner, 2001, (ASP Conf. Ser. 264); Exotic Stars as Challenges to Evolution, IAU Coll. 187, Florida, 2002, (ASP Conf. Ser.); Classical Nova Explosions, Sitges, 2002, (AIP); Symbiotic Stars Probing Stellar Evolution, La Palma, 2002, (ASP Conf. Ser.); 13th European Workshop on White Dwarfs, Naples, 2002, (Kluwer); Sixth Pacific Rim Conference on Stellar Astrophysics, Xi'an, 2002.

In addition, the following books related to close binaries appeared: An Introduction to Close Binary Stars by R. W. Hilditch and Cataclysmic Variable Stars - How and Why they Vary by C. Hellier.

\section{Eclipsing and Massive Binaries (D. Gies, N. Morrell \& V. Niemela)}

Using HST facilities, Massey et al. (2002 ApJ 565, 982) analyzed radial velocities and photometry of 4 double lined early O-type eclipsing binaries in the central cluster of 30 Doradus, the brightest HII region in the Large Magellanic Cloud. One of these objects (R136-38: O3V + O6V) has the highest stellar mass so far determined for a component of a binary system: $57 \mathrm{M}_{\odot}$. The LMC star MACHO*053441.3-693139 (Ostrov 2001 MNRAS $321, \mathrm{~L} 25$ ) is also very massive. Studies of eclipsing binaries are providing increasing evidence for a spread in distances of stars in the LMC, showing that the stellar distribution has a considerable depth (e.g. Ribas et al. 2000 ApJ 528, 692). The nature of SS Lac, an eclipsing binary that stopped eclipsing in the middle of the 20th Century, was clarified through a precise radial velocity study which discovered a third body in the system (Torres \& Stefanik 2000 AJ 119,1914 ). This might also explain other systems in which eclipses either stopped or changed significantly in depth.

There is growing evidence that the ultraluminous variable $\eta$ Car is a binary with a $5.5 \mathrm{yr}$ orbital period (Corcoran et al. $2001 \mathrm{ApJ} 547,1034$ ). It shows X-ray variations around periastron consistent with emission from colliding stellar winds (Pittard \& Corcoran 2002 A\&A 383,63), which are important for understanding the emission sources and environments of massive binaries (Moffat \& St-Louis 2002, ASP Conf. Ser. 260). The X-Mega (http://lheawww.gsfc.nasa.gov/users/corcoran/xmega/xmega.html) collaboration continued the monitoring of O-type stars known to be X-ray sources in the Carina Nebula, finding that most of them are indeed multiple systems, e.g.: CPD $-59^{\circ} 2635$ (Albacete Colombo et al. 2001 MNRAS 326, 78), CPD $-59^{\circ} 2603$ (Rauw et al. 2001 MNRAS 326, 1149). They also discovered apsidal motion for the very early O-type binary HD 93205 in the Carina Nebula (Morrell et al. 2001 MNRAS 326, 85) Several interesting papers have applied tomographic separation of composite spectra in the analysis of massive close binaries, e.g. Penny et al (1999 ApJ 518, 450; 2001 ApJ 548, 889); Harvin et al (2002 ApJ $568, \mathrm{~L} 27)$. In our Galaxy, dusty rotating pinwheels produced by WR binaries have been observed in the infrared (cf. Tuthill et al. 1999 Nature 398, 487; Monnier et al. 2002 ApJ 567, 137) and a new massive close binary with a WR type component was discovered (Niemela \& Gamen 2000 A\&A 362, 973).

For many years, high speed $O B$ runaway stars have been suspected to be the progeny of massive close binary systems, formed when one binary component explodes as a supernova, or as the result of the breakup of the binary during a close binary-binary encounter. Recent Hipparcos observations indicate both processes occur. Proper motion data show that the runaways $\mathrm{AE}$ Aur and $\mu \mathrm{Col}$ were coincident with the eccentric-orbit binary $\iota$ Ori $\sim 2.5$ Myr ago, consistent with the binary-binary encounter mechanism (Hoogerwerf et al. 2000 ApJ 544, L133; 2001 A\&A 365, 49), while the runaway $\zeta$ Oph was close to the position of the pulsar PSR J1932+1059 about $1 \mathrm{Myr}$ ago, which implicates the dissolution of a binary in a supernova. A high runaway velocity and a large eccentricity have recently been found in the young, massive X-ray binary LS 5039 (McSwain \& Gies 2002 ApJ 568, L27; Ribó et al. 2002 A\&A 384,954 ) which is also consistent with a supernova in a binary. Many of 
the massive X-ray binaries are now known to host relativistic jets and belong to the class of microquasars (Mirabel \& Rodríguez 1999 ARA\&A 37, 409).

New results on symbiotics include a new method of polarimetry which detects Raman scattering in the circumstellar gas, allowing the first determination of long (150 yr) periods for D-type symbiotics (Schmid 2001 ASP Conf. Ser. 242).

\section{RS CVn Systems (J. Linsky)}

High resolution X-ray spectroscopy with the Chandra and XMM-Newton satellites provided an important new tool for understanding coronal structure and dynamics in RS CVn systems. The analysis of these X-ray spectra is facilitated by new collisional-radiative plasma codes like APEC (Smith et al. $2001 \mathrm{ApJ}$ ) and Chianti (Landi et al. 1999 A\&AS). Brickhouse et al. (2001, ApJ) used Chandra/HETG to observe the W UMa contact system 44i Boo, where the Doppler-shift of the X-ray emission lines with orbital phase indicates that at least half of the emission is localized at high latitude. Higher velocity precision of coronal plasmas has been obtained with HST/STIS (Fe XXI $1354 \AA$ line) and FUSE (Fe XVIII $974 \AA$ line) e.g. the shape of the Fe XVIII line allowed Young et al. (2001, ApJ) to conclude that most of the emission from Capella is from the slowly-rotating G8 III star.

Simultaneous observations of RS CVn systems at X-ray, UV, and radio wavelengths have provided new insights. In their coordinated observations of $\sigma^{2} \mathrm{CrB}$ with VLA, ASCA, and RXTE, Osten et al. (2000, ApJ) showed that radio and X-ray flares are unambiguously correlated, and that during the rise phase of a large flare the plasma is hotter than $10^{8} \mathrm{~K}$. Ayres et al. (2001, ApJ) analyzed coordinated Chandra/HETG, HST/STIS, and VLA observations of HR1099. They found that coronal pressures can exceed pressures in the lower atmosphere by several orders of magnitude, but that STIS flares in the lower atmosphere often do not have X-ray counterparts, implying that these flares may be similar to solar transition region explosive events. In their analysis of a large flare in $\sigma \mathrm{Gem}$ observed simultaneously by XMM-Newton and the VLA, Güdel et al. (2002, ApJ) showed that the time derivative of the $\mathrm{X}$-ray light curve resembles the radio light curve. This phenomenon, called the "Neupert effect" for solar flares, likely results from chromospheric evaporation driven by coronal electrons when they reach the chromosphere.

Analysis of ASCA, XMM-Newton, and Chandra spectra of HR1099 (Drake et al. 2001 ApJL; Brinkman et al. 2001 A\&A), II Peg (Huenemoerder et al. 2001 ApJ), UX Ari (Güdel et al. $1999 \mathrm{ApJ}$ ), and $\sigma^{2} \mathrm{CrB}$ (Osten et al. 2000, $2002 \mathrm{ApJ}$ ) show a pattern of reduced abundances of low first ionization potential (FIP) elements like Fe and enhanced abundances of the high FIP element Ne outside of flares, but more normal abundances during flares. The He-like triplet ratios lead to coronal electron density estimates for these stars.

Pagano et al. (2001 A\&A) spectrally imaged the eclipsing AR Lac system using IUE spectra of the $\mathrm{Mg}$ II $\mathrm{k}$ line showing the location of geometrically extended structures corotating with the K0 IV star, a large cool structure near the G2 IV star, and a bright feature located near the L1 Lagrangian point that may be due to mass exchange.

\section{Contact Binaries (S. Rucinski)}

While many new W UMa type systems continue to be discovered, we still do not understand their internal structure and how these stars form and evolve. A fresh idea comes from the Stępien et al. (2001 A\&A 2001370,157$)$ postulate that all W UMa systems are semidetached binaries that just look like contact systems, but this idea requires detailed models for support. The high spatial incidence of contact binaries requires evaluation as the newest results are discordant. The OGLE and open-cluster data suggested about one contact binary per $130 \mathrm{~F}-\mathrm{G}-\mathrm{K}$ dwarfs (Rucinski $1998 \mathrm{AJ} 116,2998$ ), but this is not confirmed by the Hipparcos bright-star sample (Rucinski, astro-ph/0207144, PASP 2002), which suggests a relative frequency about 4 times smaller $(\sim 1 / 500)$, with a statistical uncertainty of a factor of about two. Either the OGLE-I material suffered from some observational problems or 
the Galactic Disk contains many more W UMa systems in its central parts than in the solar neighborhood.

\section{Cataclysmic Variables (P. Szkody)}

Three highlights stand out in the past few years. First is the discussion and variety of papers on evolution, proving that our understanding of the initial population from which CVs form, and the mechanism for the period gap are still not settled. Andronov, Pinsonneault \& Sills (2001 ApJ) applied the angular momentum loss rates of open cluster stars to infer a much longer evolution time for CVs, and a lower space density. Their derived mass-period relations imply a possibility of two populations for secondary stars that could produce the period gap. Schenker and King (2002 ASP Conf. Ser. 261) have also explored multiple evolutionary tracks with an early phase of thermal-timescale mass transfer to solve some of the discrepancies. Kolb (2002 ASP Conf. Ser. 261) points out continuing problems with the period minimum (theory predicts shorter than observed) which could disappear with different angular momentum loss rates than currently used. These ideas are strengthened by the discovery of ultrashort period systems with secondaries that are $\mathrm{K}$ stars (Thorstensen et al. $2002 \mathrm{ApJ}$ ).

The second highlight concerns the emerging consensus on the importance of magnetic fields across many areas. Warner \& Woudt (2002 MNRAS) have found that DNOs and QPOs seen in dwarf novae at outburst can be explained by a Low Inertia Magnetic Accretor Model, which assumes they are essentially low field $\left(10^{5} \mathrm{G}\right)$ IPs. They also connect many old novae with desynchronised polars. Norton et al. (2002, astro-ph 0206013) suggests the ultrashort periods seen in double-degenerates can be explained if they are IPs. The presence of circular polarization in SW Sex stars (2001 Rodriguez-Gil et al. ApJ 548, 49L) implies they are IPs. And the debate still rages as to whether some of these are magnetic propellers such as AE Aqr. Even propeller Symbiotics (fiickering systems) have been proposed to be magnetic objects.

The last highlight was the immediate and overwhelming multiwavelength observation of the rare superoutburst of WZ Sge, using Chandra, XMM, FUSE, HST and ground. The amount and quality of the data will provide fodder for outburst and disk wind theories for years to come.

\section{Surveys (S. Rucinski \& P. Szkody)}

The MACHO and OGLE micro-lensing surveys have resulted in the discovery of large numbers of binary stars, among the most interesting are three eclipsing systems containing a Cepheid as one of the components (astro-ph/0201481). After 2/3 of the 1000 eclipsing binaries in the original Galactic Bulge OGLE-I fields were identified as W UMa-type systems, the OGLE-II project increased its production rate, but most of this material remains to be analyzed. A large fraction of 68,000 variables in SMC and LMC (Zebrun et al. AcA, astro-ph/0110623) and of 200,000 variables in the Galactic Bulge (Wozniak et al. AcA, astro-ph/0201377) are expected to be eclipsing binaries.

The Hamburg Quasar Survey (HQS) covered 13,600 deg ${ }^{2}$ at high Galactic latitudes to $\sim 18.5$ mag with an objective prism. Gänsicke et al. (2002 ASP Conf. Ser. 261) conducted a systematic search for CVs and pre-CVs and found 50 new CVs. Surprisingly, followup observations of 15 showed that only one has a period below the gap. The $2 \mathrm{dF}$ survey with a multi-fiber spectrograph covers $740 \mathrm{deg}^{2}$ in two strips (one near the equator and one at the S Galactic pole). Out of $19 \mathrm{CV}$ candidates found by Marsh et al. (2002 ASP Conf. Ser. 261), orbital periods determined for four were all below the gap. The Sloan Digitial Sky Survey (SDSS) covers $7000 \mathrm{deg}^{2}$ at high Galactic latitude with 5 filter photometry to 23rd mag and multi-fiber spectroscopy of selected candidates. The first year of operation revealed 19 new CVs (Szkody et al. $2002 \mathrm{AJ}$ ) with followup observations revealing most are low mass transfer systems below the period gap. More than $100 \mathrm{WD}+\mathrm{M}$ pairs were also found. By the end of the survey, $\sim 400 \mathrm{CVs}$ and 1000 pairs are expected. The Faint 
Sky Variability Survey (FSVS; Groot et al. 2002 MNRAS) observes variability of $20 \mathrm{deg}^{2}$ in three colors to $\sim 24$ th mag. Followup on 25 out of 1200 candidates revealed two new CVs. Calàn-Tololo objective prism survey (CTS; Maza et al. 1989 ApJS 69, 349) covers $5150 \mathrm{deg}^{2}$ of the southern sky down to $\sim 18.5 \mathrm{mag}$. From a search of half of the plates, 21 CVs were found (12 new).

\section{New Missions (L. Bianci \& A. Giménez)}

In-orbit space missions will continue to provide important information for the study of close binary systems. The launch before the end of 2002 of the European high-energy mission INTEGRAL will enable a better understanding of accretion processes in $\mathrm{X}$-ray binaries. GALEX (the GALaxy Evolution EXplorer) will be launched in Jan. 2003, to perform imaging and spectroscopic surveys of the sky in the UltraViolet. GALEX will perform the first all-sky survey in two UV bands, down to $\operatorname{mag}_{A B}(2000 \AA)=21$, and deep imaging of $160 \mathrm{deg}^{2}$ down to $\operatorname{mag}_{A B}=25-26$, which is expected to reveal several thousand close binaries. GALEX will also perform slitless grism spectroscopy of the deep fields, covering $1300-3000 \AA$ with $10-20 \AA$ resolution, allowing refined temperatures and extinctions. The launch of SIRTF in early 2003 will permit new studies of the secondaries and the dust formation from ejected material. But the near future is also opening new opportunities for eclipsing binaries in the optical domain, providing light curves of unprecented precision, two orders of magnitude better than currently available from ground based observatories. These developments are linked to asteroseismology studies (MOST launch April 2003) and the search for extra solar planets through transit measurements (COROT planned for 2005 and then Kepler and Eddington in 2007). INTEGRAL also carries an optical wide-field monitor that will produce many serendipitous light curves. Thus, the future continues to be very promising for $\mathrm{C} 42$ research.

\section{Working Group on Accretion Physics in Interacting Binaries}

The working group has focussed its efforts on planning and participating in meetings on accretion across several disciplines and plans to hold two sessions devoted to accretion topics in Sydney. Accretion results are summarized quarterly in the Interacting Binaries Electronic Newsletter, which can be accessed throught the C42 website. Active researchers in the area of accretion physics are urged to join by contacting $P$. Szkody (szkody@astro.washington.edu). 